\title{
La ejecución presupuestal de ESSALUD del Perú como un instrumento de gestión ${ }^{1}$
}

\author{
ESSALUD budgetary execution in Peru as a management \\ instrument
}

\author{
Elsa Luz Yactayo Chávez²
}

\section{RESUMEN}

El modelo actual de Gestión Presupuestaria del Seguro Social de Salud en el Perú ESSALUD, según normas y procedimientos vigentes que regulan su operatividad, no está articulada a la producción de las Metas de Servicios de Salud; actividades que a pesar de estar orientados hacia un mismo objetivo no tienen un punto de convergencia, lo que limita la realización de un análisis integral del comportamiento de la producción de los servicios de salud y la utilización de los recursos presupuestales que materializan su ejecución; dificultando esta situación, la optimización de la asignación y perfeccionamiento del uso de los recursos presupuestales, y con ello, la eficiencia y la calidad de la prestación de servicios de salud. Por lo tanto, surge la necesidad de replantear el esquema presupuestal actual a un nuevo modelo presupuestario como el de Presupuesto por Resultados impulsado por el Estado actualmente. Esta alternativa corregiría los vacíos del presupuesto tradicional, mediante la articulación de Producto y Resultado, aplicando criterios de eficiencia y equidad, lo que ocasionaría cambios significativos en el manejo de la gestión presupuestal de ESSALUD.

Palabras Clave: A12 Gestión Presupuestaria y Producción Servicios de Salud; Nuevo Modelo Presupuestario.

Clasificación JEL : H11,H22,H60,H61, H75

1 Gestión Presupuestaria ESSALUD, Investigación “Ejecución Presupuestal de ESSALUD como un Instrumento de Gestión" Años 2008 - 2013. Artículo de Reflexión.

2 Economista, Universidad San Martin de Porres, Lima, Perú, Profesional Especializado, ESSALUD, Lima, Perú. Email: elsa_luz06@yahoo.com

(C) Los autores. Este artículo es publicado por Pensamiento Crítico de la Facultad de Ciencias Económicas, Universidad Nacional Mayor de San Marcos. Este es un artículo de acceso abierto, distribuido bajo los términos de la licencia Creative Commons Atribucion - No Comercia_Compartir Igual 4.0 Internacional. (http://creativecommons.org/licenses/by-nc-sa/4.0/) que permite el uso no comercial, distribución y reproducción en cualquier medio, siempre que la obra original sea debidamente citada. 


\section{SUMMARY}

The current Social Health Insurance Budgetary Management model in Peru - ESSALUD, according to current norms and procedures that regulate its operation, is not linked to the Health Services Goals production; activities which, despite being oriented towards the same objective, do not have a point of convergence, which limits the comprehensive analysis performance of the production of health services behavior and the use of budgetary resources that materialize their execution; making this situation difficult, optimizing the allocation and improving the use of budgetary resources, and with it, the efficiency and quality of the provision of health services. Therefore, there is a need to rethink the current budget structure in to a new budgetary model such as the Budget by Results, currently promoted by the State. This alternative would correct the gaps of the traditional budget, through the articulation of Product and Result, applying the efficiency and equity criteria, what will cause significant changes in budgetary management in ESSALUD.

Keywords: A12 Budgetary Management and Health Services Production; New Budgetary Model.

\section{Introducción}

El estudio examina el comportamiento de la ejecución presupuestal de ESSALUD de los años del 2008 al 2013 y destaca la importancia de la gestión presupuestaria y la permanente búsqueda por incrementar la productividad de las actividades y la utilización eficiente y racional de los limitados recursos económicos, así como al derecho que tiene el usuario final de recibir una atención satisfactoria que contribuya a mejorar su calidad de vida.

En ESSALUD, el modelo de Gestión Presupuestaria, según normas y procedimientos vigentes que regulan sus actividades, la operatividad de la fase de ejecución presupuestal no está articulada con la producción de las Metas de Servicios de Salud; situación que limita el análisis de las variables que inciden en el comportamiento de la citada producción vinculada con el aspecto presupuestal y financiero que concreta su ejecución. Consecuentemente, los datos son insuficientes para realizar evaluaciones que permitan una correcta interpretación de los problemas que se presentan en sus operaciones habituales, ante eventuales ajustes o redireccionamiento de los recursos presupuestales que deben ejecutarse en el proceso del fortalecimiento de la actividad productiva de servicios de salud. 
En este contexto, es necesario que ESSALUD, impulse cambios a efectos de otorgar una prestación de servicios de salud oportuna, eficaz y de calidad a la población asegurada. En ese sentido, la implementación de la metodología de Presupuesto por Resultados, es la reforma más importante del Estado, que tiene como fin garantizar que el usuario final reciba la atención de sus necesidades de bienes y servicios satisfactoriamente.

\section{Bases Teóricas}

\subsection{Contabilidad Administrativa 13A. Edición - Horngren, Sun- dem y Straton}

Horngren, Sundem y Straton, referente a los presupuestos y la organización, precisan que mayoría de la gente asocia la palabra presupuesto con limitaciones al gasto, y que los gobiernos suelen aprobar presupuestos de gastos para sus diversas dependencias, esperando que éstas mantengan la ejecución de sus gastos dentro del marco presupuestal aprobado. Asimismo, señala quela mayoría de las organizaciones empresariales usan los presupuestos para enfocar la atención en las operaciones presupuestales y de finanzas, no sólo para controlar el gasto, y dado que estos proporcionan información sobre los problemas potenciales y las ventajas por adelantado, igualmente lo utilizan para tomar acciones con la finalidad de evitar problemas o usar las ventajas con inteligencia oportunamente para asegurar su capacidad operativa(2006, p. 296).

Los autores indican claramente las diferencias entre el manejo de presupuestos públicos y el de los privados, precisando que el primero se caracteriza por su rigidez dado que sus operaciones presupuestales no pueden exceder el marco aprobado; en tanto que la gestión presupuestal de los privados es dinámica, ante los hechos o sucesos desfavorables ,previa evaluación de los factores que afectan el normal desarrollo de sus operaciones adoptan medidas inmediatas para su corrección, permitiendo este hecho el control de las situaciones problemáticas.

Adicionalmente, señalan quela experiencia acumulada en el manejo presupuestal de periodos pasados, es otro de los factores importante que le permite, previa evaluación y análisis de la tendencia de la ejecución del 
gasto, tomar medidas para mejorar aspectos operativos, y en base a sus objetivos para nuevos periodos plantear estrategias para que esta pueda alcanzar sus metas previstas, acciones que contribuyen al fortalecimiento de la gestión.

\subsection{Economía de la Salud: Instrumentos - Gimeno, Rubio y Tamayo. El Presupuesto en las Instituciones Públicas - Guirola y Tránchez}

Con relación al proceso de ejecución presupuestaria, afirman que en esta fase es importante la autonomía de cada centro gestor y la no interferencia del Ministerio de Publico, manteniéndose el principio de separación funciones entre el órgano competente para ordenar los pagos (centro gestor) y el encargado de la materialización de dichos pagos, de la contabilidad y de la intervención del gasto (Ministerio de Publico).

Esta ejecución de operaciones va a ser objeto de un control continuo por parte del Poder Legislativo producto de la desconfianza habitual de este poder sobre el ejecutivo. En este sentido, la mayoría de las normas presupuestarias de los otros países han regulado cuidadosamente el proceso de ejecución presupuestal del gasto durante el ejercicio presupuestario para evitar cualquier arbitrariedad. En el momento de revisar y analizar la fase de ejecución señalan que el valor jurídico del presupuesto es diferente según se refiera a los gastos o a los ingresos. (2006, p. 227).

En lo descrito, los autores destacan el aspecto de la regulación del proceso de ejecución presupuestal para evitar arbitrariedades; sobre este aspecto, es de señalar que los establecimientos hospitalarios públicos, no carecen de normas, menos de órganos institucionales que realizan el examen de control de la gestión. La práctica de estos controles están orientados a evaluar las desviaciones en el uso del presupuesto, el cumplimiento de la meta presupuestal programada y de la normatividad que lo regula, examen que además del presupuestal, comprende principalmente aspectos logísticos y contables.

No obstante se hace indispensable que las acciones de control de la ejecución presupuestal de gastos se relacione con los productos de salud, dado que mediante el uso de dichos recursos se ha concretado su realización; por lo que, es necesario proponer la implementación de un sistema 
de control interno, que contribuya al perfeccionamiento de la gestión de los hospitales vinculado al aspecto presupuestal.

\subsection{Gestión presupuestaria de estructuras: un instrumento para la gestión por resultados - Jorge Hintze}

Jorge Hintze, señala que se efectuó una encuesta a los funcionarios públicos sobre las peores características de las organizaciones públicas, obteniendo como resultado: falta de objetivos claros y la consecuente falta de responsabilidad, las mismas que técnicamente se refieren a la planificación y la organización respectivamente.

En la presentación del documento ${ }^{3}$, respecto a la estructura organizativa asevera que el presupuesto es considerado como el instrumento de planificación operativa más común para expresar la voluntad política, por lo tanto es la versión oficial disponible de la matriz de producción externa, de los recursos de la producción interna y las transformaciones organizativas.

Por otro lado, el primer nivel de estructura organizativa por lo general lo especifica como un conjunto de unidades jerárquicamente dependientes (secretarías, gerencias y direcciones). La articulación entre las estructuras presupuestaria y organizativa formalmente establecidas, se muestra usualmente como la asignación básica a determinadas unidades de la responsabilidad oficial por las metas y los recursos de determinadas categorías programáticas del presupuesto (2001, p. 19).

De acuerdo a lo anotado por el autor, el nivel de asignación descrito es básico, indicando que se requiere que este contenga un mayor detalle de las categorías programáticas del presupuesto y estas a su vez estén relacionados con los procesos de trabajo que utilizan los recursos para el logro de la meta y asimismo definir la responsabilidad de las unidades operativas y administrativas involucradas.

La referida integración de metas, recursos y funciones lo representa mediante la elaboración de matriz de corresponsabilidad, la que debe ser elaborada para cada categoría programática presupuestaria conforme lo muestra la figura que sigue (2001, p. 20):

3 Hintze, J. (2001) Documento VI Congreso Internacional CLAD. 


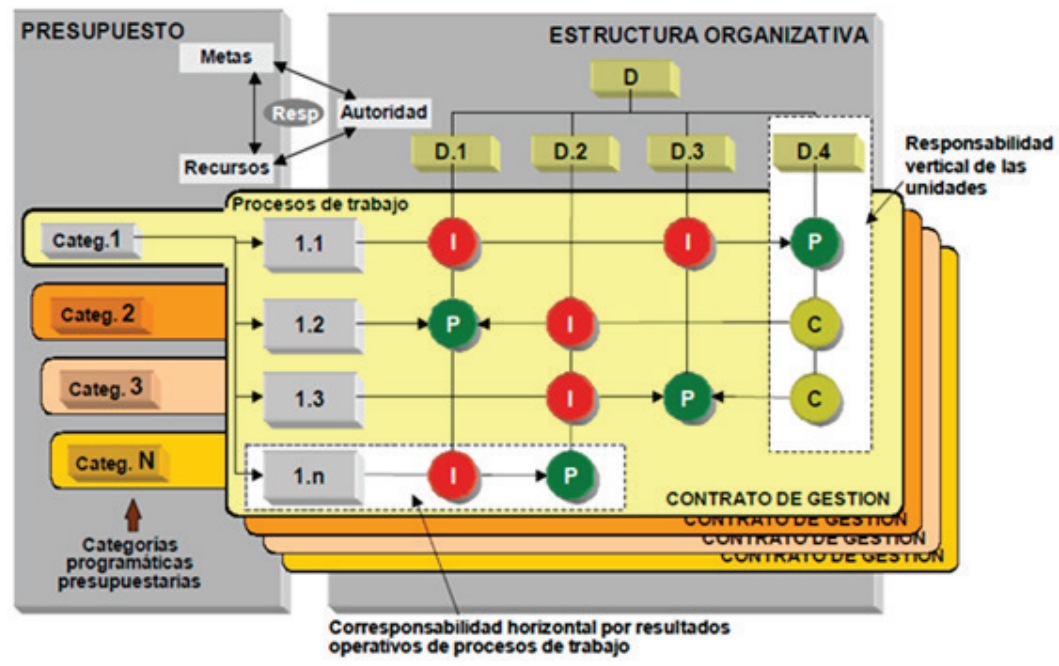

Figura $N^{\circ} 1$. Matriz presupuestaria de corresponsabilidad

\section{Mecanismos de pagos servicios de Salud}

Los mecanismos de pago son los estímulos más importantes que utilizan los gestores de salud para incentivar la conducta de los profesionales médicos o entidades intermediarias para que mejoren la producción hospitalaria y la excelencia en las atenciones médicas, así como el desarrollo de la oferta de prestación de servicios basado en la demanda, perfeccionamiento de la relación médico - paciente, integración de los profesionales médicos, entre otros. A continuación se reseñará algunos de los citados mecanismos:

\subsection{Presupuestos por Resultados PpR - Conceptos y Líneas de Ac- ción DNPP - Dirección Nacional de Presupuesto Público - Direc- ción de Economía y Finanzas - Perú - Documento de Trabajo}

El documento de trabajo de la Dirección de Economía Finanzas - Perú, respecto al Presupuesto por Resultados (PpR), sustenta que las reformas más avanzadas relacionadas con el sistema presupuestario se relacionan con el denominado PpR, catalogándolo como un instrumento característico de la nueva gerencia pública que construye las fases presupuestales en función a los resultados esperados y los bienes y servicios requeridos 
para lograrlos. Asimismo, con relación a lo anterior, precisa que el objetivo del PpR va más allá de la simple asignación de recursos públicos y control del gasto, para convertirse en una auténtica herramienta de gestión al evaluar productos y resultados relacionados con los usuarios o beneficiarios finales. (2008, p. 5, 6).

La implementación de Presupuesto por Resultados ${ }^{4}$ constituye un cambio significativo en la forma de realizar los procesos presupuestales. Representa modificar la forma tradicional de asignación presupuestal centrado en las unidades orgánicas de la institución (dependencias), programas, entre otros, estableciendo condiciones favorables en el uso de recursos al variarse el ámbito de control de los insumos a los productos y resultadoscon la finalidad de que el usuario final reciba los bienes y servicios requeridos satisfactoriamente, lo que conllevara a la mejora de su calidad de vida.

\subsection{Modelos de Pago en Servicios de Salud -Federico Tobar, Nico- lás Rosenfeld y Armando Reale}

\section{Modelo de Pago a Profesionales}

Se describirá algunos modelos que favorecen las condiciones para ofrecer una atención médica de calidad.

\section{Modelos Mixtos}

\section{a. Combinación de salario con pago por acto}

Consiste en una remuneración compuesta, parte es salario y parte por acto médico. Beneficiaría a los médicos que tratan pacientes con enfermedades crónicas.

\section{b. Pago por acto con techos individuales}

Establecimientos de techos al gasto asistencial para controlar los costos médicos, en los casos de pagos por acto médico.

\section{c. Pago por acto con techos grupales}

Consiste en la fijación de tope sobre el total de pagos a todos los profesionales, asimismo, establece para los casos que el total del

4 Ley № 28927, Ley de Presupuesto del Sector Publico para el año Fiscal 2007. 
servicio médico exceda el tope fijado se reducirá el pago por unidad de atención.

\section{- Métodos de Pagos a Instituciones}

Este método, en el marco institucional, constituye modalidades de financiamiento, lo que hace necesario diferenciar entre el modelo de financiamiento de la oferta del modelo de la demanda.

\section{Financiamiento de la oferta}

Con referencia a este modelo los autores mencionan que desde mediados de los ochenta ha adquirido peso en el sector la propuesta de la Public Competition (competición dentro del sector público) que plantea fortalecer la función del Estado como comprador antes que reformar el financiamiento global de los sistemas. Por ello reemplazan el financiamiento realizado a través del presupuesto tradicional por modelos articulados a la producción.

Modelos:

a. Pago por prestaciones

b. Pago por día (Per Diem)

c. Capitación

\subsection{Remuneración a los proveedores de servicios de salud en Bogotá, Luis G. Morales-Sánchez y Juan C.Garcia-Ubaque}

Hay diversos métodos para mejorar las relaciones financieras entre aseguradores y proveedores de los servicios de salud, entre los más relevantes están:

a. Reembolso de los servicios ofrecidos a la población asegurada atendida en hospitales autogestionados, además porque conducirá a un aumento de los ingresos institucionales, así como una mejora en la equidad del sistema de financiamiento de la salud.

b. Pagar a los médicos para la coordinación de la atención de determinadas enfermedades de interés. 
c. Agrupación de pagos por episodio o enfermedad.

d. Modelos contractuales de riesgo y utilidad compartida entre proveedores y pagadores con acuerdos más o menos formales de ahorro compartido, participación en las ganancias y pérdidas y alianzas bajo el enfoque de "Alta confianza y baja burocracia"

\section{Metodología}

\section{Alcance}

El tipo de investigación utilizada es aplicada y la información es de fuente primaria. En este estudió se aplicó el método descriptivo, que se utiliza para recoger, organizar, resumir, analizar y generalizar los resultados de las observaciones realizadas, y en mérito a dicha labor presentar una alternativa para establecer un mecanismo tangible y mensurable de evaluación de los resultados y performance de la Institución.

Sujeto del estudio: ESSALUD es el sujeto del estudio.

\section{Población de Estudio}

No tiene una población de estudio, el ámbito del estudio alcanza a todo el Seguro Social de Salud y dentro de ella a todas sus dependencias principalmente a las Redes Asistenciales Centros e Institutos Especializados que brindan la prestación de Servicios de Salud, del periodo 2008 - 2013.

\section{Tratamiento de los datos}

A partir de los datos obtenidos se aplica la Técnica de Observación, que es el método fundamental de obtención del dato de la realidad, mediante la percepción intencionada y selectiva de un objeto o de un fenómeno determinado y toma de información para su posterior análisis.

El estudio se ha orientado a la observación y análisis de los datos de ejecución de las metas de asistenciales y los gastos presupuestales que demandaron su ejecución en ESSALUD en los años del 2008 al 2013. Teniendo en cuenta el amplio número de metas (productos) asistenciales que esta entidad ofrece a la población asegurada en sus establecimientos hospitalarios y detalle de las partidas presupuestales; el análisis y la ob- 
servación del comportamiento de la ejecución de la producción de salud se efectúo por familias de productos, igualmente se procesó los datos de la ejecución rubros presupuestales.

ESSALUD, en el cumplimiento de sus funciones esenciales además de las actividades de prestaciones de salud, desarrolla actividades de prestaciones sociales y económicas, sobre lo cual se ofrece una visión general de los recursos presupuestales que gestiona de la referida Institución.

\section{Evolucion de ejecución presupuestal ESSALUD años 2008 -2013}

\subsection{Ingresos:}

En ESSALUD, las partidas componentes del rubro de ingresos son: aportaciones, prestaciones a no asegurados, ingresos financieros y otros; siendo el de mayor relevancia el concepto de aportaciones, cuya recaudación ha representado una participación del 96.5\% de la captación de ingresos institucionales anuales, la misma que ha mostrado un crecimiento promedio de $10.2 \%$ en los periodos anuales de estudio; En tanto, que en los últimos años, ante un menor crecimiento de la actividad económica, se pronosticó un comportamiento conservador, debido a la desaceleración de la economía peruana por la reducción de exportaciones, debilidad de la inversión, entre otros. A continuación se muestra la evolución de la ejecución presupuestal de ingresos de los años 2008 - 2013 expresado en valores nominales:

Tabla № 01

Evolucion ejecucion presupuestal de ingresos ESSALUD años 2008 - 2013 (En Millones de Soles)

\begin{tabular}{|c|c|c|c|c|c|c|c|c|c|c|c|}
\hline \multirow[b]{2}{*}{ RUBROS } & \multicolumn{6}{|c|}{ EJECUCIÓN ANUAL } & \multicolumn{5}{|c|}{ VARIACION ANUAL (\%) } \\
\hline & 2008 & 2009 & 2010 & 2011 & 2012 & 2013 & $\begin{array}{c}2009 / \\
2008\end{array}$ & $\begin{array}{c}2010 / \\
2009\end{array}$ & $\begin{array}{c}2011 / \\
2010\end{array}$ & $\begin{array}{l}2012 \text { / } \\
2011\end{array}$ & $\begin{array}{c}2013 / \\
2012\end{array}$ \\
\hline INGRESOS & $5,177.6$ & $5,683.0$ & $6,073.2$ & $6,390.3$ & $7,385.7$ & $8,261.9$ & $9.8 \%$ & $6.9 \%$ & $5.2 \%$ & $15.6 \%$ & $11.9 \%$ \\
\hline De Operación & $5,177.6$ & $5,683.0$ & $6,023.9$ & $6,390.3$ & $7,385.7$ & $8,259.8$ & $9.8 \%$ & $6.0 \%$ & $6.1 \%$ & $15.6 \%$ & $11.8 \%$ \\
\hline De Capital & - & - & - & - & - & 2.1 & $\cdot$ & $\cdot$ & $\cdot$ & $\cdot$ & $\cdot$ \\
\hline Financiamento Neto & - & - & 49.2 & - & - & - & - & - & $-100 \%$ & - & - \\
\hline
\end{tabular}

Fuente: Gerencia Presupuesto - ESSALUD

\subsection{Egresos:}

Gasto de personal, comprende el pago regular de las remuneraciones y obligaciones sociales de los profesionales de la salud (personal 
médico, técnicos, auxiliares asistenciales, entre otros) y administrativos, gasto que reflejó un incremento anual promedio de 9.5\% en los años 2008 $-2013$.

Compra de bienes, comprende principalmente la compra de material estratégico (medicina, material médico, material e insumos de laboratorio, material radiológico y ropa hospitalaria) que ha representado en promedio el 91.4\% del monto asignado al citado rubro en el periodo estudio.

Servicios Prestados por Terceros, comprende los servicios complementarios entre los que figuran lavandería y alimentación, limpieza y vigilancia, entre otros para garantizar el funcionamiento óptimo de los establecimientos hospitalarios; así como servicios de salud contratados a privados para atender la mayor demanda de la población asegurada.

Detalle de ejecución presupuestal de egresos de los años 2008 - 2013 expresado en valores nominales:

Tabla № 02

Evolucion ejecucion presupuestal de egresos ESSALUD años 2008 - 2013 (En Millones de Soles)

\begin{tabular}{|c|c|c|c|c|c|c|c|c|c|c|c|}
\hline \multirow[b]{2}{*}{ RUBROS } & \multicolumn{6}{|c|}{ EJECUCIÓN ANUAL } & \multicolumn{5}{|c|}{ VARIACION ANUAL (\%) } \\
\hline & 2008 & 2009 & 2010 & 2011 & 2012 & 2013 & $\begin{array}{c}2009 / \\
2008\end{array}$ & \begin{tabular}{|c|}
$2010 /$ \\
2009
\end{tabular} & $\begin{array}{c}2011 / \\
2010\end{array}$ & $\begin{array}{c}2012 / \\
2011\end{array}$ & $\begin{array}{c}2013 / \\
2012\end{array}$ \\
\hline EGRESOS & $5,177.6$ & $5,683.0$ & $6,072.9$ & $6,290.8$ & $6,835.7$ & $7,666.8$ & $9.8 \%$ & $6.9 \%$ & $3.6 \%$ & $8.7 \%$ & $12.2 \%$ \\
\hline De Operación & $4,589.1$ & $5,156.7$ & $5,590.0$ & $5,880.8$ & $6,297.5$ & $7,287.3$ & $12.4 \%$ & $8.4 \%$ & $5.2 \%$ & $7.1 \%$ & $15.7 \%$ \\
\hline De Capital & 588.5 & 526.3 & 482.9 & 410.0 & 538.2 & 379.5 & $-10.6 \%$ & $-8.2 \%$ & $-15.1 \%$ & $31.3 \%$ & $-29.5 \%$ \\
\hline
\end{tabular}

Fuente: Gerencia Presupuesto - ESSALUD

\section{Prestaciones Servicios de Saludorganos Desconcentrados - ESSALUD años 2008 - 2013}

\subsection{Producción de Servicios de Salud}

La asignación presupuestal de los Órganos Desconcentrados (años 2008 - 2013) se ha definido mediante la aprobación de metas de salud anuales y la aplicación de los Mecanismos de Asignación de Fondos Presupuestales para las Prestaciones de Salud ${ }^{5}$ que comprende: Mecanismo de asignación de recursos presupuestarios capitado (per cápita) para

5 Directiva de Gerencia General № 19-GG-ESSALUD - 2008 “ Lineamientos Generales para los Mecanismos de Asignación de Fondos Presupuestales para las Prestaciones Asistenciales” 
centros de primer nivel de atención (atención primaria) y Mecanismo de asignación de recursos presupuestarios por producción de actividades médicas, quirúrgicas, para centros asistenciales de mayor nivel hospitalario y especializados.

Tabla № 03

Evolucion produccion de servicios de salud por grupos de familias ESSALUD años 2008 2013. En miles de Unidades Fisicas

\begin{tabular}{|c|c|c|c|c|c|c|c|c|c|c|c|c|}
\hline \multirow{2}{*}{ FAMILIAS DE PRODUCTOS } & \multicolumn{6}{|c|}{ EJECUCIÓN ANUAL } & \multirow{2}{*}{$\begin{array}{c}\text { VARIACION } \\
\text { ACUMULADA } \\
2013 / 2008\end{array}$} & \multicolumn{5}{|c|}{ VARIACION ANUAL (\%) } \\
\hline & 2008 & 2009 & 2010 & 2011 & 2012 & 2013 & & 2009/2008 & $2010 / 2005$ & 9 2011/201 & 0 2012/2011 & 2013/2012 \\
\hline Prestaciones Ambulatorias & $24,661.6$ & $30,338.1$ & $34,901.4$ & $38,340,3$ & 38,3621 & $39,266.7$ & $59.2 \%$ & $23.0 \%$ & $15.0 \%$ & $9.9 \%$ & $0.1 \%$ & $2.4 \%$ \\
\hline - Consutas Médicas & $14,362.9$ & $17,373.9$ & $19,408.2$ & $20,357.4$ & $18,020.9$ & $18,805.1$ & $30.9 \%$ & $21.0 \%$ & $11.7 \%$ & $4.9 \%$ & $-11.5 \%$ & $4.4 \%$ \\
\hline - Otras Prestaciones Ambulatonias & $10,298.7$ & $12,964.2$ & $15,493.2$ & $17,982.9$ & $20,341.2$ & $20,461.6$ & $98.7 \%$ & $25.9 \%$ & $19.5 \%$ & $16.1 \%$ & $13.1 \%$ & $0.6 \%$ \\
\hline Servicios de Hosptalización & $2,251.6$ & $2,379.1$ & $2,711.6$ & $2,798.2$ & $2,831.2$ & $2,916.6$ & $29.5 \%$ & $5.7 \%$ & $14.0 \%$ & $3.2 \%$ & $1.2 \%$ & $3.0 \%$ \\
\hline Servicios de Diấisis & 600.9 & 628.8 & 658.5 & 704.5 & 733.7 & 746.7 & $24.3 \%$ & $4.6 \%$ & $4.7 \%$ & $7.0 \%$ & $4.1 \%$ & $1.8 \%$ \\
\hline Emergencia & $3,937.5$ & $4,306.5$ & $4,589.7$ & $4,813.1$ & $5,458.8$ & $5,835.9$ & $48.2 \%$ & $9.4 \%$ & $6.6 \%$ & $4.9 \%$ & $13.4 \%$ & $6.9 \%$ \\
\hline Partos & 79.0 & 86.0 & 90.0 & 93.2 & 97.7 & 105.6 & $33.7 \%$ & $8.8 \%$ & $4.7 \%$ & $3.6 \%$ & $4.8 \%$ & $8.2 \%$ \\
\hline Intervenciones Quirúrgicas & 272.6 & 311.8 & 324.8 & 316.4 & 276.7 & 295.2 & $8.3 \%$ & $14.4 \%$ & $4.2 \%$ & $-2.6 \%$ & $-12.5 \%$ & $6.7 \%$ \\
\hline Procedmientos & $3,850.1$ & $4,729.9$ & $5,133.3$ & $5,800.3$ & $6,129.7$ & $11,705.1$ & $204.0 \%$ & $22.9 \%$ & $8.5 \%$ & $13.0 \%$ & $5.7 \%$ & $91.0 \%$ \\
\hline Tratamientos Especializados & 511.5 & 539.5 & 671.1 & 779.4 & 775.9 & 826.6 & $61.6 \%$ & $5.5 \%$ & $24.4 \%$ & $16.1 \%$ & $.0 .4 \%$ & $6.5 \%$ \\
\hline Atención Domialiana & 327.4 & 338.4 & 347.8 & 360.0 & 3623 & 380.4 & $16.2 \%$ & $3.4 \%$ & $2.8 \%$ & $3.5 \%$ & $0.6 \%$ & $5.0 \%$ \\
\hline Otros Servicios " & 307.6 & 323.6 & 304.8 & 318.6 & 338.8 & & $-100.0 \%$ & $5.2 \%$ & $5.8 \%$ & $4.5 \%$ & $6.3 \%$ & $-100.0 \%$ \\
\hline Apoyo al Diagnostico & $35,317.0$ & $37,003.7$ & $39,222.3$ & $45,273.3$ & $47,028.3$ & $51,530.1$ & $45.9 \%$ & $4.8 \%$ & $6.0 \%$ & $15.4 \%$ & $3.9 \%$ & $9.6 \%$ \\
\hline Servicios Contraados & 598.1 & 673.5 & $1,594.1$ & $1,739.6$ & $1,810.6$ & $1,795.9$ & $200.3 \%$ & $12.6 \%$ & $136.7 \%$ & $9.1 \%$ & $4.1 \%$ & $-0.8 \%$ \\
\hline
\end{tabular}

Fuente: Evaluación Presupuestal Anual / Gerencia de Presupuesto - ESSALUD * Otros servicios registrado en Otras Prestaciones Ambulatorias en el año 2013

- Prestaciones Ambulatorias, este producto presentó una significativa reducción de sus niveles de ejecución alcanzando un avance solo de $0.1 \%$ en el 2012, respecto al año 2011, presentando la mayor participación en este resultado, la ejecución negativa del producto consulta médica (11.5\%).

- Hospitalización, la mayor variación positiva corresponde al año 2010 (14.0\%) y un bajo nivel de avance al año 2012 (1.2\%); asimismo, en algunas dependencias como las Redes Asistenciales Amazonas y Huancavelica la producción del año 2013 es menor respecto a la del 2008 .

- Intervención Quirúrgica, presentó variaciones negativas en los años $2011(-2.6 \%)$ y $2012(-12.5 \%)$ respecto a su similar del año anterior. Comparada la producción del 2013 con la del 2008, la variación acumulada fue de solo el $8.3 \%$. 


\subsection{Ejecución Presupuestal Egresos Operativos Órganos Des- concentrados}

La Ejecución Presupuestal de egresos operativos de los Órganos Desconcentrados de ESSALUD, se plantea a partir de la operativización del Plan Operativo Institucional, en el cual, está comprendido la Programación de Metas de Prestaciones de Salud y la asignación de recursos presupuestales. El desarrollo de esta fase demanda la ejecución de gastos en los rubros de remuneraciones, bienes y servicios para efectivizar la realización de las metas señaladas. La ejecución presupuestal de egresos operativos de los Órganos Desconcentrados de ESSALUD ha presentado una variación acumulada del 65.2\% en el periodo de estudio 2008- 2013, representado una tendencia creciente del gasto, cuyo detalle por rubro de gastos (en valores nominales) se aprecia en el siguiente cuadro:

Tabla № 04

Evolucion ejecucion presupuestal organos desconcentrados ESSALUD años 2008 - 2013 (En Millones de Soles)

\begin{tabular}{|c|c|c|c|c|c|c|c|c|c|c|c|c|}
\hline \multirow[b]{2}{*}{ DEPENDENCIAS } & \multicolumn{6}{|c|}{ EJECUCION ANUAL } & \multirow{2}{*}{$\begin{array}{l}\text { VARIACION } \\
\text { ACUMUL. } \\
2013 / 2008\end{array}$} & \multicolumn{5}{|c|}{ VARIACION ANUAL (\%) } \\
\hline & 2008 & 2009 & 2010 & 2011 & 2012 & 2013 & & $\begin{array}{c}2009 / 200 \\
8\end{array}$ & \begin{tabular}{|c|}
$2010 / 200$ \\
9
\end{tabular} & \begin{tabular}{|c|}
$2011 / 201$ \\
0
\end{tabular} & \begin{tabular}{|c|}
$2012 / 201$ \\
1
\end{tabular} & $\begin{array}{c}2013 / 201 \\
2\end{array}$ \\
\hline Total Egresos Operativos & 3,293 & 3,753 & 4,052 & 4,309 & 4,620 & 5,439 & $65.2 \%$ & $14.0 \%$ & $8.0 \%$ & $6.3 \%$ & $7.2 \%$ & $17.7 \%$ \\
\hline Gasto de Personal & 1,955 & 2,151 & 2,290 & 2,462 & 2,657 & 3,226 & $65.0 \%$ & $10.0 \%$ & $6.5 \%$ & $7.5 \%$ & $7.9 \%$ & $21.4 \%$ \\
\hline Compra de Bienes & 825 & 968 & 1,074 & 1,079 & 1,176 & 1,360 & $64.9 \%$ & $17.4 \%$ & $10.9 \%$ & $0.5 \%$ & $9.0 \%$ & $15.7 \%$ \\
\hline Servicios Prestado $\times$ Tercero: & 512 & 634 & 688 & 768 & 788 & 852 & $66.3 \%$ & $23.7 \%$ & $8.5 \%$ & $11.6 \%$ & $2.6 \%$ & $82 \%$ \\
\hline
\end{tabular}

Fuente: Gerencia Presupuesto - ESSALUD

\subsection{Comparativo: Producción Metas Asistenciales VS Gastos Pre- supuestales}

La producción de las metas asistenciales de ESSALUD muestra un incremento acumulado de $59.3 \%$ al año 2013 respecto al 2008, en tanto que para el mismo periodo el incremento de la ejecución de egresos presupuestales fue del 65.2\%; comparada las referidas informaciones muestran una diferencia del 5.9\%; resultado, al que solo se le puede dar una interpretación general, dado que los elementos que intervienen, según la metodología aplicada, resultan insuficientes para realizar un análisis y evaluación de los factores económicos y/o sanitarios que tuvieron incidencia en dicho comportamiento. 
Tabla № 05

Comparativo: Producción Servicios de Salud vs Ejecucion PPT al años 2008-2013. Organos Desconcentrados

\begin{tabular}{|c|c|c|c|c|c|c|c|c|c|}
\hline \multirow{2}{*}{ DESCRIPCION_FAMILIA } & \multicolumn{2}{|c|}{$\begin{array}{c}\text { PRODUCCION } \\
\text { (En Miles Unidades Fisicas) }\end{array}$} & \multicolumn{2}{|c|}{ VARIACION } & \multirow{2}{*}{$\begin{array}{l}\text { RUBROS } \\
\text { GASTOS }\end{array}$} & \multicolumn{2}{|c|}{$\begin{array}{c}\text { EJECUCION } \\
\text { (En Miles de Soles) }\end{array}$} & \multicolumn{2}{|c|}{ VARIACION } \\
\hline & 2008 & 2013 & En Unidades & En \% & & 2008 & 2013 & En S/. & En \% \\
\hline PRESTACIONES AMBULATORIAS & $24,661.6$ & $39,266.7$ & $14,605.1$ & 59.2 & & & & & \\
\hline -Consultas Médicas & $14,362.9$ & $18,805.1$ & $4,442.2$ & 30.9 & & & & & \\
\hline - Otras Prestaciones Ambulatorias & $10,298.7$ & $20,461.6$ & 10,1628 & 98.7 & GASTODE & 19549671 & 32250686 & 12710015 & 650 \\
\hline SERVICIO DE HOSPITALIZACION & $2,251.6$ & $2,916.6$ & 665.0 & 29.5 & PERSONAL & $1,904,90 /, 1$ & $3,220,908.0$ & $1,2 / 1,001.5$ & 00.0 \\
\hline SERVICIO DE DIALISIS & 600.9 & 746.7 & 145.8 & 24.3 & & & & & \\
\hline EMERGENCIA & $3,937.5$ & $5,835.9$ & $1,898.4$ & 48.2 & & & & & \\
\hline PARTOS & 79.0 & 105.6 & 26.6 & 33.7 & & & & & \\
\hline INTERVENCIONES QUIRURGICAS & 272.6 & 295.2 & 226 & 8.3 & & & & & \\
\hline PROCEDIMENTOS & $3,850.1$ & $11,705.1$ & $7,855.0$ & 204.0 & COMPRA & & & & \\
\hline MEDICINA COMPLEMENTARIA & 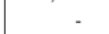 & 404.1 & 404.1 & 0.0 & DE BIENES & $824,856.8$ & $1,360,481.0$ & $535,624.2$ & 64.9 \\
\hline TRATAMENTOSESPECIALIZADOS & 511.5 & 826.6 & 315.1 & 61.6 & & & & & \\
\hline ATENCION DOMICILIARIA & 327.4 & 380.4 & 53.0 & 16.2 & & & & & \\
\hline OTROS SERVICIOS & 307.6 & - & 307.6 & -100.0 & & & & & \\
\hline APOYO AL DIAGNOSTICO & $35,317.0$ & $51,530.1$ & $16,213.1$ & 45.9 & SERVICIOS & $512,873.9$ & $852,118.7$ & $339,244.7$ & 66.1 \\
\hline SERVICIOS CONTRATADOS & 598.1 & $1,795.9$ & $1,197.8$ & 200.3 & & & & & \\
\hline TOTAL & $72,714.8$ & $115,808.8$ & $57,699.1$ & 59.3 & TOTAL & $3,292,697.8$ & $5,438,568.3$ & $2,145,870.4$ & 65.2 \\
\hline
\end{tabular}

Fuente: Evaluación Presupuestal Anual, Gerencia de Presupuesto - ESSALUD Elaboración propia

\section{Resultados}

Hipótesis: La ejecución presupuestal del Seguro Social de Salud, influye positivamente como un verdadero instrumento de gestión institucional de la entidad en estudio.

La Hipótesis fue evaluada, analizada e interpretada tomando en cuenta las siguientes variables:

- Variable Independiente: Ejecución Presupuestal

- Variable Dependiente: Instrumento de Gestión Institucional

De lo expresado, se aprecia que los avances anuales muestran una razonable ejecución presupuestal, sin embargo en la realidad en los establecimientos hospitalarios se observa un descontento de los asegurados en lo que respecta a una adecuada y oportuna atención médica, debido principalmente a los problemas de citas prolongadas, las colas, insuficiencia de médicos especializados, sobre stocks y desabastecimiento de algunos bienes, entre otras necesidades.

Entre las causas de esta situación, está relacionado con los modelos de Gestión Presupuestaria y de Prestaciones de Servicios de Salud que se 
aplican en ESSALUD, los que no tienen un punto de convergencia, a pesar de orientarse hacia un mismo objetivo. En el aspecto presupuestal, la programación, ejecución y control presupuestal está ligada al establecimiento asistencial pero no articulado al producto de salud final, esto último permitiría, por ejemplo, definir la razonabilidad del volumen de las compras de los diferentes tipos de material estratégico con referencia a la programación de metas de salud, evitando los sobre stock o desabastecimiento; lo que evitaría la distracción de los escasos recursos económicos.

\section{Prueba de Hipótesis}

En la investigación se identificó apropiadamente las variables de la realidad, tanto independiente como dependiente, las cuales se contrastó adecuadamente, concretándose que la variable independiente la constituye la "Ejecución Presupuestal", del seguro social de la entidad en salud, y a su vez la considera un verdadero instrumento de gestión institucional, de la entidad en estudio. Esto corrobora que los hechos observados concuerdan con la hipótesis planteada.

\section{Discusión de la investigacion}

Los resultados de la investigación son coherentes desde el planteamiento del problema, es decir nuestros planteamientos teóricos en materia de ejecución presupuestal, en que se anota que debe ser eficiente, eficaz y transparente, pero que en la realidad en los años materia de estudio no ha ocurrido con tal eficacia, afectando así la gestión institucional por falta de implementación una nueva metodología de gestión presupuestaria que corrija las deficiencias en el aprovisionamiento de recursos humanos y materiales en relación a las necesidades reales de los asegurados. Finalmente los resultados guardan relación con la hipótesis de la investigación a la entidad en estudio.

De acuerdo a lo indicado, los mecanismos de pago son estímulos que utilizan los gestores de salud para incentivar la conducta de los profesionales médicos o entidades intermediarias a fin de conseguir mejoras principalmente en la productividad y la calidad de las atenciones médicas ,oferta de prestación de servicios, relación médico - paciente, entre otros. Sin embargo no existe un arreglo financiero que pueda definirse categóri- 
camente como apropiado; este debería definirse según la realidad de cada caso y los objetivos pretendidos.

Por otro lado, la Revista de Salud Publica ${ }^{6}$ plantea que la eficiencia técnica (provisión de servicios de salud al menor costo) y locativa (optimización de la mezcla de los servicios de salud) es un punto crítico de cualquier sistema de salud. Pero dada las distorsiones intrínsecas (limitaciones de respuesta de los proveedores y pobre capacidad de los pacientes para expresar sus preferencias), el mercado de servicios de salud por sí mismo no logra corregirlas, por tanto es importante promover la educación de los pacientes (empoderamiento) y de los médicos (uso de guías de práctica clínica)y facilitar las condiciones para estimular el mejor uso de los recursos y eliminar incentivos perversos

\section{Conclusiones}

- La ejecución presupuestal del Seguro Social de Salud, es muy importante después de la programación, en realidad constituye un verdadero instrumento de gestión institucional de la entidad, pero siempre que se realice con eficiencia, eficacia y transparencia en bien de los asegurados.

- Los resultados de la ejecución presupuestal de los años 2008 al 2013, no son satisfactorios para los asegurados, no obstante la entidad gasta al $100 \%$ de lo presupuestado, pues hay deficiencia en el aprovisionamiento de material estratégico y en la calidad de atención al paciente asegurado, así como falta de médicos especializados entre otras carencias.

- La entidad del Seguro Social de Salud cuenta con el Órgano de Control Institucional, como el apoyo de la Contraloría General de la República, para fiscalizar el cumplimiento de políticas en materia presupuestal. La dependencia institucional solo evalúa la razonabilidad de la gestión presupuestaria desvinculado de la producción de metas asistenciales, actividades que generaron el gasto.

6 Remuneración a los proveedores de servicios de salud en Bogotá Luis G. Morales-Sánchez y Juan C.Garcia-Ubaque 


\section{Recomendaciones}

- La Alta Dirección de ESSALUD, en relación a las políticas presupuestales, a través de los órganos competentes, debe evaluarla implementación de mecanismos de pago siendo uno de ellos el de presupuesto por resultados, para así alcanzar con eficacia el objetivo primario de la Institución, la atención integral de los asegurados.

- El órgano de control institucional de la entidad, como la Contraloría General de la Republica, deben evaluar con mayor eficacia la ejecución presupuestal en armonía con los objetivos del Plan Operativo Institucional y la implementación del presupuesto por resultados.

- La entidad del Seguro Social de Salud, debe cambiar las formas tradicionales inerciales, al momento presupuestar deben existir criterios lógicos, implementando los mecanismos de pago, mejorando el planeamiento estratégico, los controles organizacionales (políticas), como mejorar el control interno por resultados en bien de los asegurados de la entidad en estudio.

\section{Referencias Bibliográficas}

Dirección Nacional de Presupuesto Público - Ministerio de Economía y Finanzas. "Conceptos y Líneas de Acción" (Documento de Trabajo) DNPP -, Edición Diciembre 2008. Recuperado en https//www.mef-gob.pe/contenidos/presu$\mathrm{publ} / \mathrm{ppr} /$ conceptos-lineas-accion.pdf

Gimeno, J.,Rubio, S. y Tamayo, P. (2006). Manuales de Dirección Médica y Gestión Clínica "Economía de la Salud: Instrumentos". España. Ediciones Díaz de Santos

Hintze, J. (2001). Documento presentado como ponencia en el VI Congreso Internacional del CLAD sobre Reforma del Estado y la Administración Pública. "Gestión Presupuestaria de Estructuras: un instrumento para la gestión por resultados" Buenos Aires, Argentina.

Horngren, Ch. - Sundem, G .- Straton W. (2006) "Contabilidad Administrativa". México. 13A. Edición. 720p 
Morales-Sánchez L, Garcia-Ubaque J. (2017). Remuneración a los proveedores de servicios de salud en Bogotá. Revista de Salud Pública. 19 (2), 219-226. Recuperado en http://www.scielo.org.co/pdf/rsap/v19n2/0124-0064-rsap-19-02-00219.pdf

Tobar F, Rosenfeld. M. y Reale, A.(1989). Modelo de Pago de Servicios en Salud. Cuadernos Médicos Sociales $\mathrm{N}^{\circ} 74$. Recuperado en file://C:/Users/Director/ Downloads/Modelos_de_Pago_en_servicios_de_salud.pdf

Seguro Social de Salud - ESSALUD. Planeamiento Estratégico 2003 - 2007. Perú. Recuperado en Web: www.essalud.gob.pe/transparencia/pdf/ planes/4pestrategico.pdf

Seguro Social de Salud - ESSALUD. Planeamiento Estratégico 2012 - 2016. Perú. Web: http://www.essalud.gob.pe/transparencia/pdf/planes/ plan_2012_2016.pdf

Ley № 28927, Ley de Presupuesto del Sector Público para el Año Fiscal 2007. Artículo 10․- De la Implementación del Presupuesto por Resultados (PpR). El Peruano. Lima, 12 diciembre 2006.

Ley № 29289, Ley de Presupuesto del Sector Público para el Año Fiscal 2009. Artículo 79․- Del Presupuesto por Resultados (PpR). El Peruano. Lima, 11 diciembre 2008. 